OPEN ACCESS

Edited by:

Fan Yi,

Shandong University, China

Reviewed by:

Dongshan Zhang,

Central South University, China

Ding-Sheng Jiang

Huazhong University of Science and

Technology, China

${ }^{*}$ Correspondence:

Chunlin Ou

ouchunlin@csu.edu.cn

†These authors have contributed equally to this work

Specialty section:

This article was submitted to Nephrology,

a section of the journal

Frontiers in Medicine

Received: 05 November 2020 Accepted: 25 March 2021

Published: 20 April 2021

Citation:

He X, Kuang G, Zuo Y, Li S, Zhou S and Ou C (2021) The Role of

Non-coding RNAs in Diabetic Nephropathy-Related Oxidative Stress. Front. Med. 8:626423.

doi: 10.3389/fmed.2021.626423

\section{The Role of Non-coding RNAs in Diabetic Nephropathy-Related Oxidative Stress}

\author{
Xiaoyun $\mathrm{He}^{1+}$, Gaoyan Kuang ${ }^{2 t}$, Yi Zuo ${ }^{3+}$, Shuangxi $\mathrm{Li}^{4}$, Suxian $\mathrm{Zhou}^{3}$ and Chunlin $\mathrm{Ou}^{1,5 *}$ \\ ${ }^{1}$ Department of Pathology, Xiangya Hospital, Central South University, Changsha, China, ${ }^{2}$ Department of Orthopedics, The \\ First Affiliated Hospital of Hunan University of Chinese Medicine, Changsha, China, ${ }^{3}$ Department of Endocrinology, Affiliated \\ Hospital of Guilin Medical University, Guilin, China, ${ }^{4}$ Department of Pathophysiology, Hunan University of Medicine, Huaihua, \\ China, ${ }^{5}$ National Clinical Research Center for Geriatric Disorders, Xiangya Hospital, Central South University, Changsha, \\ China
}

Diabetic nephropathy (DN) is one of the main complications of diabetes and the main cause of diabetic end-stage renal disease, which is often fatal. DN is usually characterized by progressive renal interstitial fibrosis, which is closely related to the excessive accumulation of extracellular matrix and oxidative stress. Non-coding RNAs (ncRNAs) are RNA molecules expressed in eukaryotic cells that are not translated into proteins. They are widely involved in the regulation of biological processes, such as, chromatin remodeling, transcription, post-transcriptional modification, and signal transduction. Recent studies have shown that ncRNAs play an important role in the occurrence and development of DN and participate in the regulation of oxidative stress in DN. This review clarifies the functions and mechanisms of ncRNAs in DN-related oxidative stress, providing valuable insights into the prevention, early diagnosis, and molecular therapeutic targets of DN.

Keywords: diabetic nephropathy, oxidative stress, ncRNA, mircoRNA, therapeutic target

\section{INTRODUCTION}

Diabetic nephropathy (DN) is one of the main complications of diabetes mellitus (DM) and the main cause of diabetic end-stage renal disease (ESRD), resulting in the disability and death of patients with DM $(1,2)$. DN is characterized by progressive renal interstitial fibrosis, which is accompanied by a series of pathological changes, including excessive accumulation of extracellular matrix (ECM) components, thickening of the glomerulus and tubular basement membrane, and increased formation of glomerular and tubular basement membrane matrix $(3,4)$. The increased prevalence of DM has led to an increase in the incidence of DN. DN is a leading cause of ESRD $(5,6)$, which is one of the major health problems worldwide.

The imbalance between oxidants and antioxidants is called oxidative stress (OS) and occurs when the body is subjected to various harmful stimuli, leading to the injury of tissue and cells (7). OS exists in all stages of DM development, and hyperglycemia is the main factor promoting OS (8-12). In addition, the advanced glycation end products (AGEs) associated with hyperglycemia (13), reactive oxygen species (ROS) $(14,15)$, the protein kinase C (PKC) pathway (16), and the renin-angiotensin system promote the occurrence of OS (17) and its maintenance, and cause the 
development of DN (8). Furthermore, the abundance of mitochondria in kidney tissue renders it more vulnerable to OS (18). Intrarenal OS, which also plays a vital role in the pathogenesis of DN, causes chronic inflammation of the kidney, and glomeruli and tubular hypertrophy (19).

Ribonucleic acid (RNA) is divided into two categories according to its characteristics: coding RNA and non-coding RNA (ncRNA). Research on ncRNAs has demonstrated that these molecules are not simply "junk" transcription products but functional regulatory molecules that mediate cellular processes. Many ncRNAs affect specific cellular biological responses, and are key regulatory molecules in the course of disease (20). For example, small ncRNAs, such as, microRNAs (miRNAs), may act as proto-oncogenes or tumor suppressor genes in cancers $(21,22)$; circular RNAs (circRNAs) participate in the development of tumors, neurological diseases, and rheumatic diseases (23-25); small nucleolar RNAs (snoRNA) participate in tumors, metabolic stress, and other diseases through modification $(26,27)$; long-chain ncRNAs (lncRNAs) have been linked to cancer, diabetes, heart failure, hypertension, kidney disease, and other diseases (28-32). Thus, ncRNAs are a new hot spot in epigenetic research. Although, the role of several ncRNAs in disease pathogenesis has been revealed, their specific regulatory networks have yet to be studied.

\section{CLASSIFICATION OF ncRNAs}

The Human Genome Project led researchers to discover that protein-coding sequences only account for nearly $2 \%$ of the human genome, while the remaining non-coding regions were considered "junk areas." The development of computational biology and the popularization of genome sequencing technology showed that these "junk regions" are transcribed into large amounts of RNA, of which nearly $74 \%$ are ncRNAs $(33,34)$. According to their functions and sizes, ncRNAs are roughly divided into three categories: housekeeping ncRNAs, small RNAs (sRNAs), and lncRNAs; circRNAs constitute a special type of lncRNA. Housekeeping ncRNAs are essential for cellular activities, and include ribosomal RNAs (rRNAs), transfer RNAs (tRNAs), small nuclear RNAs (snRNAs), and snoRNAs (35). sRNAs generally refer to ncRNAs shorter than 200 nucleotides (nt). According to their species origin, they are divided into two categories: bacterial sRNAs and eukaryotic sRNAs. Eukaryotic sRNAs include miRNAs, small interfering RNAs (siRNAs), and PIWI-interacting RNAs (piRNAs). lncRNAs have lengths of more than $200 \mathrm{nt}$ (36), and are widely transcribed in the genome. According to their transcriptional location, molecular characteristics, or position relative to mRNAs, lncRNAs are divided into long intervening/intergenic ncRNAs, which are located in the gap between two mRNAs and have an independent transcription; natural antisense transcripts, which reversely overlap with an mRNA exon; promoter upstream transcripts (prompts); enhancer RNAs, which are transcribed from enhancers of protein-coding genes; and circRNAs $(37,38)$.

\section{BIOLOGICAL CHARACTERISTICS OF ncRNAs}

ncRNAs are widely involved in important biological functions, such as, the development and differentiation of cell development and differentiation, reproduction, cell apoptosis, and cell reprogramming, and are closely associated with disease development and progression. To date, many studies on miRNAs, lncRNAs, and circRNAs have been conducted (Figure 1).

\section{miRNA Function}

It is generally believed that miRNAs bind to RNA-induced silencing complexes in the cytoplasm, recognizing, and binding their matching target sequence (usually in the $3^{\prime}$ untranslated region of the protein-coding gene) in a sequence-specific manner, thus, regulating the degradation of target gene mRNA and/or its translation to inhibit gene expression. However, some studies have suggested that some miRNAs, such as miR-320, miR373, miR-122, and miR-483, may also promote or inhibit the expression of target genes in the nucleus (39). Nuclear miRNAs may recognize and bind target sequences in gene promoters and other DNA regulatory elements in a sequence-specific manner, and then recruit proteins or complexes responsible for epigenetic modification, which results in chromatin remodeling, resulting in transcriptional activation or gene silencing. Alternatively, nuclear miRNAs inhibit lncRNAs near target genes, thereby regulating gene expression (40). At present, the reasons, mechanisms, and functions of miRNA accumulation in the nucleus remain unclear and require further investigation. Based on the role of miRNAs in regulating target gene expression, the functions of miRNAs are determined by the functions of their target genes. If the target genes regulate cell proliferation, apoptosis, differentiation, and other important biological functions, the miRNAs will play an important role in these biological functions. miRNAs have obvious cell line specificity in the regulation of target genes. Different miRNAs in the same cell line have different roles, and the same miRNAs in different cell lines also have different functions. Hundreds of miRNAs in cells have a complex regulatory role in tens of thousands of protein-coding genes, forming a genome-wide expression regulatory network that keeps protein expression at normal levels. Hence, miRNA abnormality often affects important physiological processes and triggers the occurrence of major diseases. Studies have shown that the abnormal regulation of miRNAs is a common feature of many diseases (41), making them a novel interventional target. Due to the stability of miRNAs in organisms, their application in the diagnosis and prognosis of disease will be useful. In short, miRNAs have tremendous potential in disease treatment and translational medicine.

\section{IncRNA Function}

lncRNAs are linear ncRNA transcripts with complex structures, and are widely expressed in mammalian genomes. Compared with mRNA, lncRNAs have low expression levels, high tissue specificity, and strong temporal and spatial expression specificity (42). It is estimated that more than 10,000 lncRNAs exist 


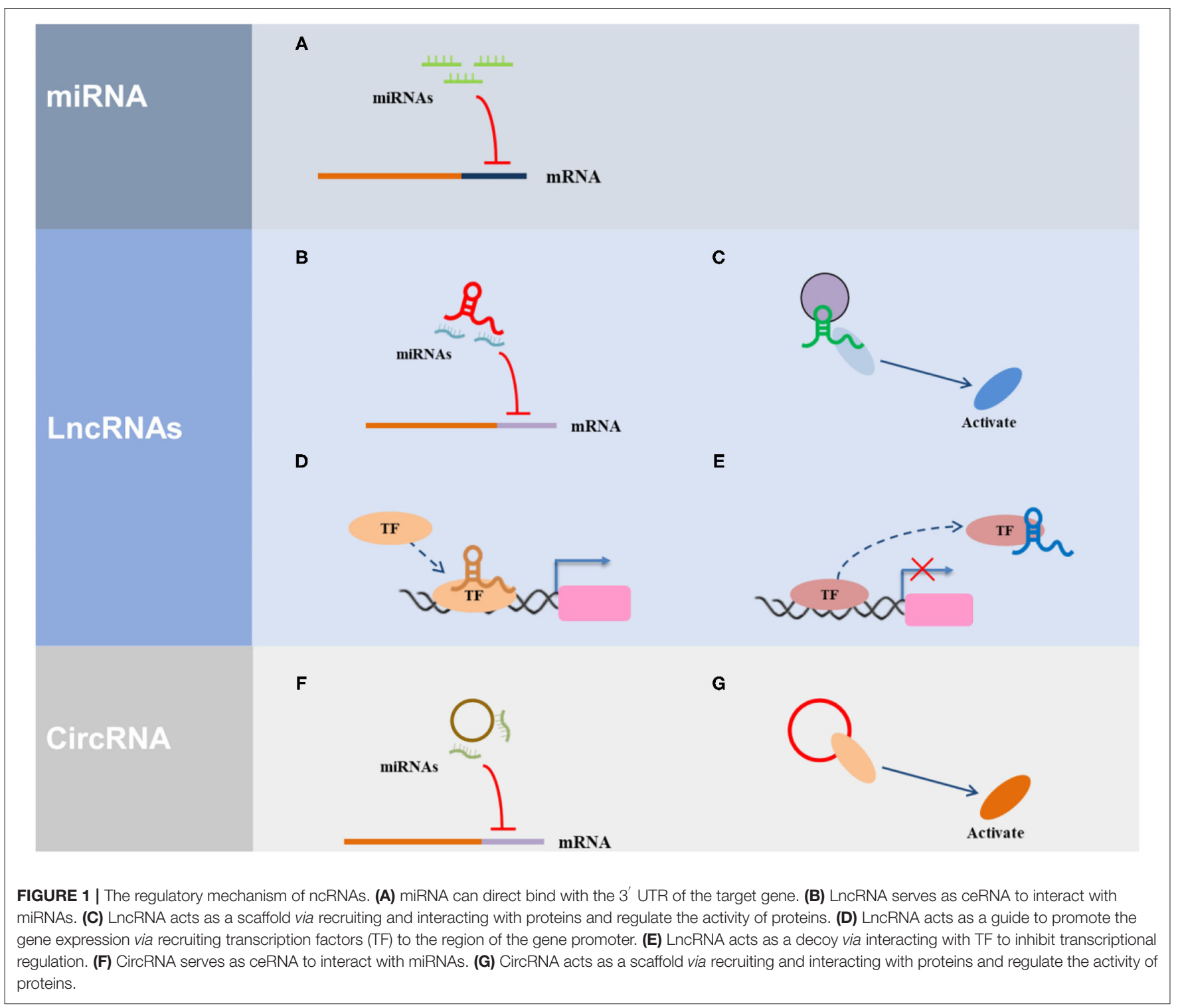

in the human genome. However, biochemical identification and functional research is still in its infancy. At present, the functions of only $\sim 100$ lncRNAs are understood. These lncRNAs regulate the expression or activity of target genes at the DNA, RNA, and protein levels, and are widely involved in a variety of important regulatory processes, such as, the inactivation of the $\mathrm{X}$ chromosome, genomic imprinting, stem cell pluripotency, somatic cell reprogramming, chromatin remodeling, the formation of nuclear substructures, and intranuclear transport, and are associated with the occurrence and development of many human diseases, such as, tumors, cardiovascular diseases, and neurological diseases (43-47). lncRNA regulation of target protein-coding genes occurs both during and after transcription, as lncRNAs may be located in the nucleus or cytoplasm. It has been shown that $30 \%$ of all $\operatorname{lncRNAs}$ are located in the nucleus, $15 \%$ in the cytoplasm, and the rest are found in both the nucleus and the cytoplasm.

Recently, with increasing research and the development of sequencing technology, an increasing number of lncRNAs and functions have been reported. Currently, there are over 20,000 lncRNA annotations, which is more than protein-coding gene annotations. For the whole genome of a cell, the expression of lncRNAs is much lower than that of protein-coding genes, and exhibits obvious tissue and cell line specificity. Some lncRNAs begin to function at certain stages of eukaryotic development. Because lncRNAs regulate the expression of protein-coding genes in different ways, lncRNAs are involved in many important biological processes, including heredity, development, cell cycle, and changes in chromosome structure. IncRNA 
abnormalities are involved in the development of many common diseases $(45,47,48)$.

\section{circRNA Function}

circRNAs are special ncRNAs that form closed loop structures through covalent bonds $(49,50)$. circRNAs are believed to mainly exist in the cytoplasm, with a small amount found in the nucleus $(51,52)$. circRNA expression is extremely abundant in eukaryotes and is evolutionarily conserved. Although, they do not encode proteins, they interact with proteins, regulate the variable splicing process of pre-mRNA, and regulate the maturation of rRNAs $(53,54)$. In addition, circRNAs play an indispensable role in the normal physiological processes of biological reproduction, growth, and aging, and are involved in the occurrence and development of neurological diseases, autoimmune diseases, cardiovascular diseases, and tumors $(23,55)$. Some circRNAs regulate the expression of protein-coding genes by competitively binding miRNAs (56). Furthermore, both lncRNAs and circRNAs are used as competitive endogenous RNAs to combine with miRNAs, forming an interactive regulatory network (57). The development of bioinformatics technology will allow further understanding of the functional role of these three types of ncRNAs, which will help us clarify the pathogenesis of certain diseases and develop therapeutic strategies and drugs.

\section{Other ncRNAs Function}

Other ncRNAs mainly contain snoRNAs, which can be divided into three categories based on their structural elements: box C/D snoRNA, box H/ACA snoRNA, and MRP RNA $(58,59)$. The main snoRNAs in cells are box $C / D$ and box H/ACA snoRNAs. In addition, snoRNAs can be classified based on their gene organization, into independently coding snoRNA and intron coding snoRNA. snoRNAs can be classified based on their gene organization, ranging from independently transcribed genes under the control of independent promoters, to intronic coding units, which lack an independent promoter and are encoded in introns of protein-coding host genes. snoRNA participates in the biosynthesis of eukaryotic ribosomes, mainly to guide the modification of nucleotides at specific sites and participate in rRNA shearing $(59,60)$. Cajal body-specific small RNA (scaRNA) is similar to snoRNA and has box C/D or boxH/ACA structural elements (61). scaRNA guides the nucleotide modification of snRNA. Moreover, a special class of molecules including U85, U87, U88, U89, with both box $\mathrm{C} / \mathrm{D}$, and box H/ACA domains were found, which can simultaneously guide the ribose methylation and pseudouracilization of U5 and U4 snRNA. Vertebrate telomerase is a box H/ACA telomerase box, which may be essential for in vivo telomerase accumulation, 3 ' end processing of the telomerase RNA precursor to ensure the stability of mature RNA, and telomerase activity. Mutations in human telomerase box H/ACA motif or the bound small nucleolar ribonucleoprotein (snoRNP) dyskerin cause multisystem genetic diseases (62). Although, some small RNAs have the typical structure of box C/D or box H/ACA, and primarily guide the chemical modification of other RNAs, including rRNAs, tRNAs, and snRNAs, a large subclass of snoRNAs called orphan snoRNAs cannot find complementary sequences that match rRNA or snRNA (63). It has been reported that many RNAs that are not directly related to ribosomal biosynthesis, including a small number of mRNAs, can temporarily stay in the nucleolus. Moreover, in yeast, the RNAse P-mediated $5^{\prime}$ end processing of some tRNA precursors occurs in the nucleolus. Therefore, it is possible that orphan snoRNAs act on RNAs other than rRNA and snRNA.

\section{DN PATHOGENESIS}

$\mathrm{DN}$ is characterized by pathological albumin excretion or albumin/creatinine ratio in the urine of patients with diabetes and a decrease in the glomerular filtration rate (64-66). Pathological changes in DN, such as, glomerulus enlargement, basement membrane thickening, and accumulation of glomerular mesangial and tubular ECM, lead to glomerular and tubular interstitial fibrosis, and even hardening $(67,68)$. $\mathrm{DN}$ is the main cause of ESRD worldwide and is related to the incidence and mortality of cardiovascular events $(8,69,70)$. Risk factors for the occurrence and development of DN include increased inflammation, oxidation markers, AGEs, ROS, elevated levels of transforming growth factor- $\beta$ (TGF- $\beta$ ), elevated PKC levels, abnormal polyol metabolism, uric acid levels, a long history of DM, age at diagnosis, race, systemic or glomerular hypertension, proteinuria, genetic susceptibility, insulin resistance, and diet composition $(69,71)$.

The pathogenesis of $\mathrm{DN}$ is complex process, involving in a series of signaling pathway changed (Figure 2). Moreover, the pathogenesis of DN depends on the following aspects: (1) Genetic susceptibility factors. Specific single nucleic acid polymorphisms in susceptibility genes have been associated with DN, and therefore, research on this area is helpful for the prevention of DN (72-74); (2) Abnormal glucose metabolism. Hyperglycemia promotes the occurrence of a variety of pathophysiological processes, including the activation of the polyol pathway (75, 76) and the generation and accumulation of AGEs (77-79); (3) Inflammatory reactions. DN is an inflammatory disease caused by a metabolic disorder As such, inflammatory reactions accompany the entire process of DN development, which result in the gradual scarring of the renal glomeruli, known as glomerulosclerosis (67, 80, 81); (4) Cytokines. Proinflammatory cytokines affect hemodynamics, promote cell proliferation, and increase ECM secretion and renal interstitial fibrosis, thus participating in the occurrence and development of DN (82); (5) OS. Excessive ROS production in the body activates PKC and the polyol pathway, leading to an increase in AGEs, and cytokine release, eventually resulting in severe pathological changes in the kidneys and promoting the occurrence and development of DN (83); (6) Endoplasmic reticulum stress (ERS). Excessive ERS may cause OS by promoting an increase in ROS, thereby damaging the kidneys (83-85) and participating in the development of DN; (7) Autophagy. This is the process whereby damaged proteins and organelles are decomposed after a stress response, and this plays an important role in maintaining cell homeostasis (86). The activation of the mechanistic target of rapamycin (mTOR) (87, 88 ) and the reduction of $5^{\prime}$ AMP-activated protein kinase (89) 


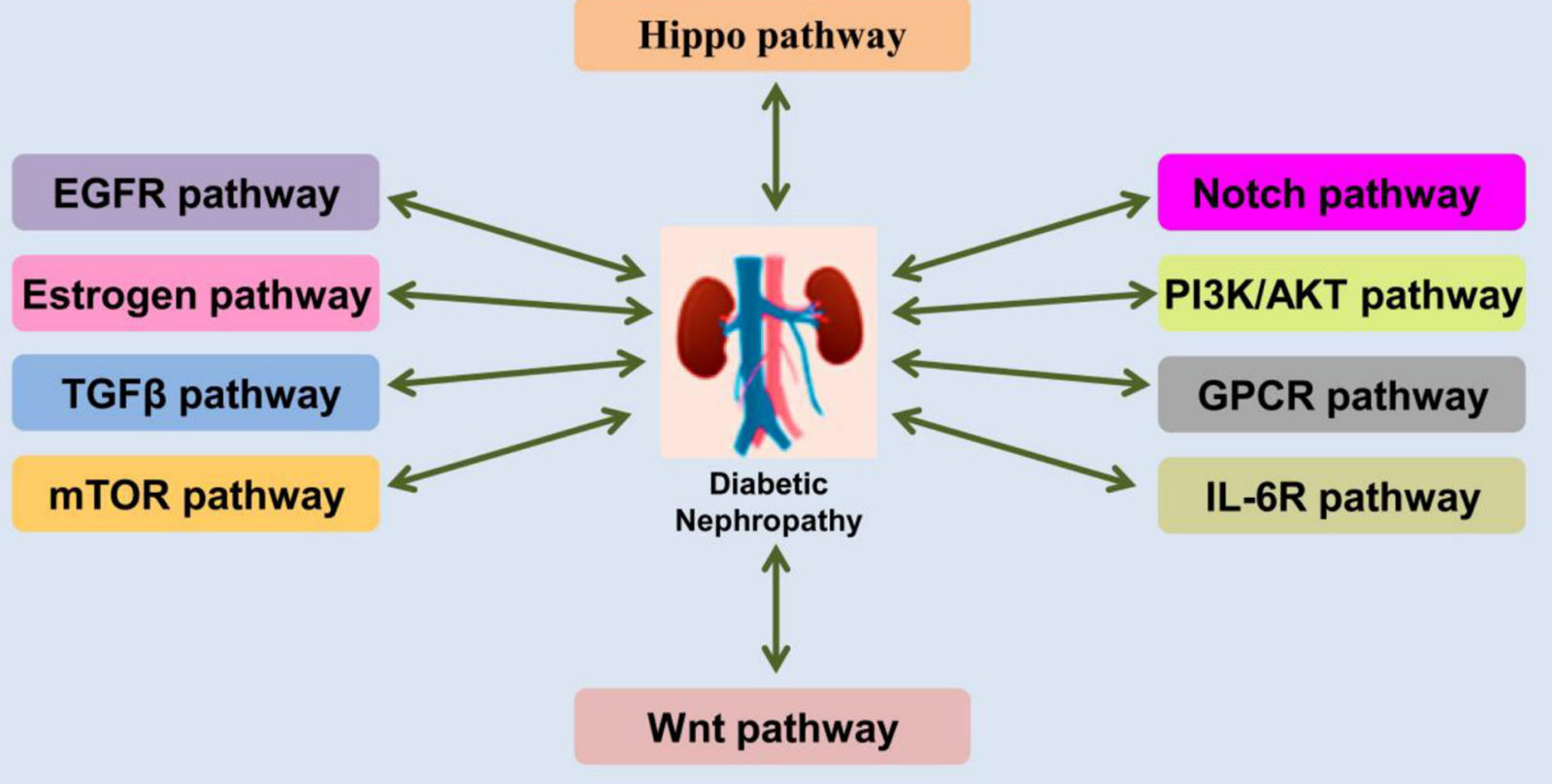

FIGURE 2 | Schematic demonstration of the crosstalks between DN and signaling pathways.

and Sirtuin 1 (SIRT1) (90) attenuate autophagy-related activities, and this attenuation has been linked to the pathogenesis of DN; (8) Exosomes and extracellular vesicles. Recently discovered, exosomes and extracellular vesicles are closely related to the occurrence and development of DN. The expression of exosomes is abnormal in DN, and the DNA, RNA, and protein contained in them are involved in the pathogenesis of $\mathrm{DN}$ and are used as molecular markers of $\mathrm{DN}(91,92)$. In general, the pathogenesis of $\mathrm{DN}$ is complex, and what is currently known may just be the tip of the iceberg. Therefore, the integration of multiple aspects of the disease is crucial for the development of effective treatments.

Previous studies have reported that oxidative stress plays an important role in the occurrence and development of DN (3, 93-95). Hyperglycemia induces renal cells to produce large amounts of ROS, which increases oxidative stress. During oxidative stress, multiple signal pathways such as, glucose oxidation, production of advanced glycation end products (AGE), activation of protein kinase, hexosamine, and polyol pathways are involved in the metabolic regulation of glucose and lipids (96). Oxidative stress in the kidneys usually results in the massive production of ROS by peroxidase, which induces renal fibrosis and inflammation, and leads to tissue injury by promoting lipid peroxidation, DNA damage, and mitochondrial dysfunction (97). Under normal physiological conditions, ROS play a significant role in the regulation of cell proliferation, differentiation, apoptosis, and immune defense, while under the pathological conditions of diabetes, excessive ROS production in the kidneys stimulates the recruitment of inflammatory cells and the release of large amounts of inflammatory factors, growth factors, and transcription factors (71), thereby altering kidney structure and function, and promoting DN (98). Oxidative stress also accelerates the occurrence and development of DN by damaging the podocytes in the glomerular filtration barrier. The mechanisms whereby oxidative stress induces podocyte injury include ROS-induced mitochondrial dysfunction, activation of the mitogen-activated protein kinase (MAPK), and NF- $\mathrm{B}$ signaling cascade, and oxidative damage of DNA (99). In addition, oxidative stress contributes to the development of glomerulosclerosis. ROS activate signaling pathways such as angiotensin II/transforming growth factor $\beta 1$ (TGF- $\beta 1$ )/smad, protein kinase $\mathrm{C}(\mathrm{PKC})$, and $\mathrm{NF}-\kappa \mathrm{B}$, inducing the deposition of extracellular matrix. However, signaling factors such as angiotensin II, TGF- $\beta 1$, and PKC also facilitate the generation of ROS, aggravating DN and oxidative stress damage. Moreover, oxidative stress is involved in the development of renal tubular fibrosis. ROS stimulate the expression of a variety of pro-fibrotic growth factors such as TGF- $\beta 1$, vascular endothelial growth factor (VEGF), and connective tissue growth factor, further boosting the deposition of extracellular matrix proteins and renal function damage (100). Therefore, it is extremely crucial to explore the mechanisms whereby oxidative stress participates in the occurrence of DN.

\section{RELATIONSHIP BETWEEN ncRNAs AND DIABETIC NEPHROPATHY-RELATED OS}

In recent years, various studies have confirmed that ncRNAs participate in the occurrence and development of $\mathrm{DN}$ by regulating OS. Among the ncRNAs that regulate OS in $\mathrm{DN}$, 

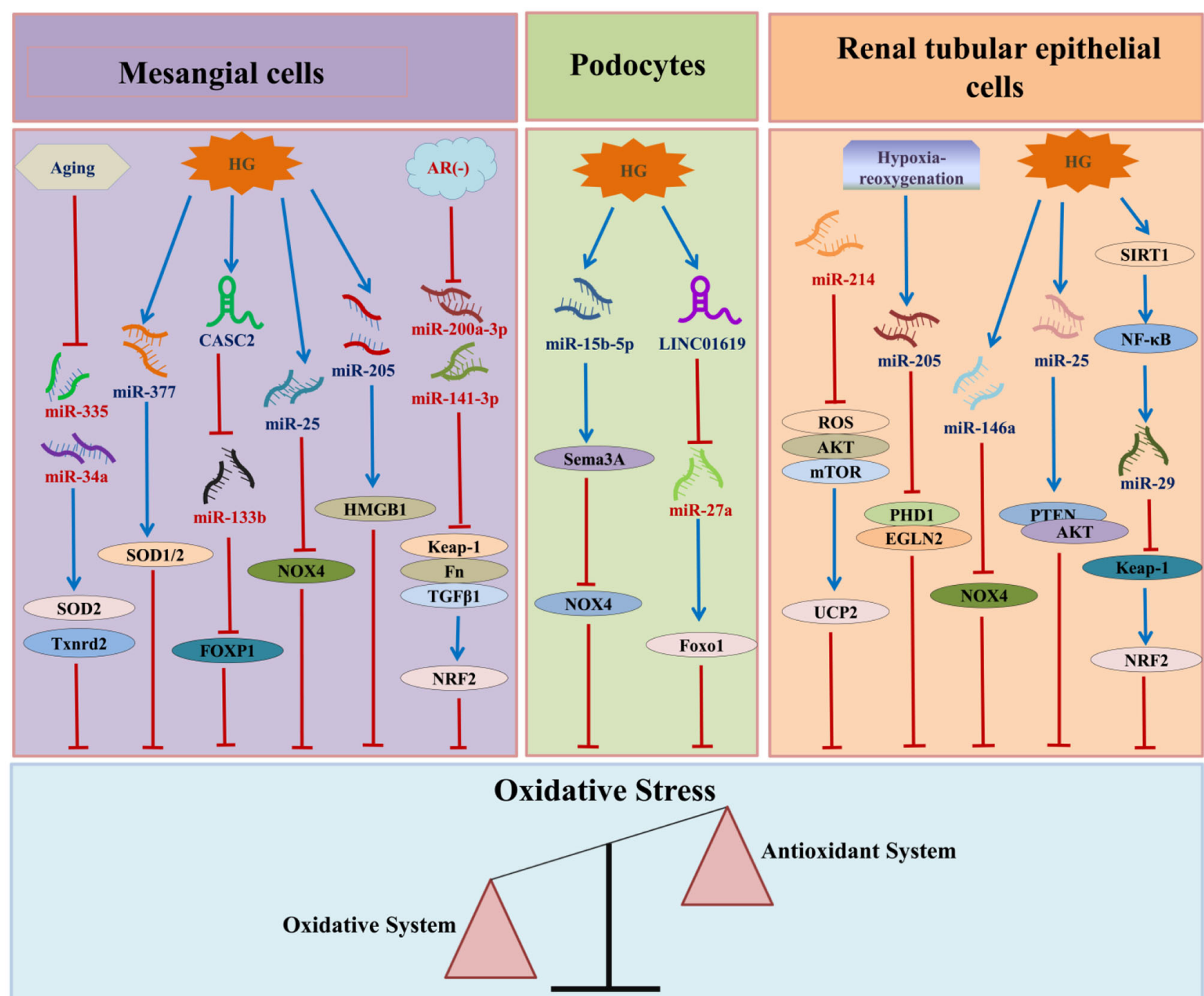

FIGURE 3 | The relationship between ncRNAs and DN-associated oxidative stress. HG, high glucose; AR, aldose reductase; CASC2, cancer susceptibility candidate 2; HMGB1, high mobility group box 1; SOD, superoxide dismutase; NOX4, nicotinamide adenine dinucleotide phosphate oxidase 4; FN, fibronectin;

TGF- $\beta 1$ :transforming growth factor $\beta 1$ :TXNRD2, thioredoxin reductase 2; FOXP1, forkhead box P1; NRF2, nuclear factor erythroid E2-related factor 2; SEMA3A, semaphorin 3A; Foxo1, Forkhead box 1; ROS, reactive oxygen species; AKT, protein kinase B; mTOR, mechanistic target of rapamycin; UCP2, uncoupling protein 2; SIRT1, Sirtuin 1; NF-кB, nuclear factor-kappa B; PTEN, phosphatase and tensin homolog; PHD1, prolyl hydroxylase 1.

miRNAs are the most widely studied, followed by lncRNAs and circRNAs (Figure 3).

\section{miRNAs and Diabetic \\ Nephropathy-Related OS}

miRNAs are post-transcriptional regulatory RNAs with a length of 18-23 nt that are widely present in eukaryotes, do not encode proteins, and are able to inhibit gene expression through specific interactions with target genes. Altered miRNA expression levels may generate OS and ultimately result in the development of disease. The relationship between miRNA and OS in the pathogenesis of DN has also become a research hotspot in recent years.

\section{Up-Regulated miRNAs}

The miR-23a/27a/24-2 cluster upregulates c-jun N-terminal kinases (JNKs) to induce caspase-dependent and caspaseindependent cell death in human embryonic kidney cells (HEKT293), which is accompanied by an increase in ROS (101). In addition, overexpression of the miR-23a/27a/24-2 cluster results in changes in HEKT293 ERS and mitochondrial membrane permeability (102), suggesting that the cluster is closely related to OS in the kidney. Uncoupling protein 2 (UCP2), a negative regulator of ROS generation (103), is the target gene of miR-24 in the kidney; The downregulation of hsa-miR-24-3p results in UCP2 upregulation and subsequent reduction in ROS production (104). Shao et al. (105) found that miRNA-3550 is 
upregulated in the kidneys of DN rats and is related to the $\mathrm{Wnt} / \beta$ catenin signaling pathway. miR-27a directly targets nuclear factor erythroid 2-related factor 2 (NRF2), interfering with ROS homeostasis in $\mathrm{DN}$, while adipokinin omentin 1 upregulates NRF2, and reduces OS by inhibiting miR-27a, restoring kidney function in type 2 diabetic $\mathrm{db} / \mathrm{db}$ mice (106).

miRNA-452-5p expression is increased in high glucose (HG)treated HK-2 cells (a renal tubular epithelial cell line), which is accompanied by an in increase in ROS and malondialdehyde (MDA) levels, and a decrease in SOD levels (107). However, these changes are reversed by interfering with miR-452-5p activity (108), which suggests that miR-452-5p is involved in the HGinduced OS response of renal tubular cells. Compared to controls, the levels of microRNA-377 have been shown to be consistently up-regulated in in vitro and mouse diabetic nephropathy models. The activity of miR-377 leads to reduced expression of p21activated kinase and superoxide dismutase, which enhances fibronectin (FN) production. Thus, the overexpression of miR377 in diabetic nephropathy indirectly leads to increased FN production. In addition, miR-377 targets SOD1 and SOD2 in human mesangial cells (HMCs) (109). Bioinformatics analysis and verification of the miRNA expression profiles in kidneys of young and old rats demonstrated that mitochondrial Sod2 and thioredoxin reductase 2 (Txnrd2) are the targets of miR335 and miR-34a, respectively. In aging MCs, miR-335 and miR-34a are significantly upregulated, while Sod2 and Txnrd2 are significantly downregulated, which is consistent with the production of ROS (110). Kato et al. (111) showed that TGF$\beta$ activates protein kinase B (AKT) in glomerular mesangial cells by inducing miR-216a and miR-217, leading to glomerular mesangial cell proliferation and hypertrophy. Zhang et al. (112) found that miR-133b is upregulated in the serum and HMCs of patients with $\mathrm{DN}$, and that miR-133b targets forkhead box P1 (FOXP1). The upregulation of lncRNA CASC2 inhibits HGinduced HMC proliferation, ECM accumulation, and OS via the miR-133b/FOXP1 regulatory axis. Podocyte damage is a sign of $\mathrm{DN}$, and is induced via ERS by the upregulation of miR-27a, which negatively targets forkhead box 1 (FOXO1) (113).

After analyzing miRNAs in the serum of healthy controls, patients with type $2 \mathrm{DM}$, and patients with DN, Regmi et al. (114) found that serum miR-99b and miR-122 levels are significantly increased in DM and DN group patients, while those of miR-20a, and miR-486 are decreased, and that the levels of these miRNAs are significantly related to albuminuria, glomerular filtration rate, blood sugar, and blood lipid levels. Moreover, target gene prediction of these four miRNAs revealed that they regulate OS, inflammation, and apoptosis (114). The emergence of RNAseq technology has facilitated the discovery of miRNAs related to OS in DN and the identification of their target genes, enabling in-depth research on the pathogenesis of DN.

\section{Down-Regulated miRNAs}

Renal tubular epithelial cells are one of the main cells that absorb glucose; however, long-term hyperglycemia directly causes their damage, and induces dysfunction through OS. In HG-treated HK-2 cells, the deacetylase activity of SIRT1 is weakened, resulting in a decrease in NF- $\kappa \mathrm{B}$ activity. NF- $\kappa \mathrm{B}$ regulates the expression of miR-29, which targets KEAP1 by directly binding to its promoter. Downregulation of miR-29 in response to HG enhances the expression of KEAP1, and reduces NRF2 levels through ubiquitination, thereby inducing the damage of renal tubular epithelia (115). Moreover, miR-29 expression is negatively correlated with serum creatinine levels and creatinine clearance in diabetic rats (115). Yang et al. (116) found that miR214 inhibits $\mathrm{OS}$ in DN and enhances the expression of UCP2 through the ROS/AKT/mTOR signaling pathway in HK-2 cells. The expression of UCP2 attenuates mitochondrial ROS activity, thereby exerting an antioxidant effect.

Superoxide derived from nicotinamide adenine dinucleotide phosphate oxidase (NOX) plays a key role in hyperglycemiaderived OS in DN; OS production by NOX mediates matrix accumulation and renal fibrosis in DN $(117,118)$. In HG-treated MCs and the kidneys of streptozotocin (STZ)-induced diabetic rats, the expression of miR-25 is significantly reduced, while the mRNA and protein levels of NOX4 are increased (119). MCs transfected with antagomiR-25 showed a considerable increase in the mRNA and protein levels of NOX4 (120). These results are consistent with the increased OS and diastolic dysfunction observed in the hearts of hypercholesterolemic rats when the expression of miR-25 is decreased (121). Thus, the miR-25NOX4-OS axis seems to play a common role in kidney and heart diseases. In addition, miR-25 is downregulated in kidney biopsy tissue and serum of patients with $\mathrm{DN}$, and is inversely proportional to proteinuria. Moreover, HG-treated HK-2 cells show decreased miR-25 levels in a time-dependent manner. Overexpression of miR-25 reduces the generation of ROS in HK-2 cells; the mechanism may be related to the activation of the phosphatase and tensin homolog/AKT pathway (122). Similarly, Wan et al. (123) confirmed that miR-146a expression is inhibited and NOX4 levels are increased in a DN mouse model. In addition, overexpression of miR-146a in HK-2 cells inhibits NOX4 expression, reducing ROS production, OS, and inflammation levels (123), which suggests that miR-146a has an anti-inflammatory and oxidation modulating effect in DN.

Wei et al. (124) reported that aldose reductase (AR) negatively regulates the expression of miR-200a-3p/miR-141-3p in MCs. In STZ-induced diabetic mice, AR deficiency significantly increases the miR-200a-3p/miR-141-3p levels in the renal cortex, which is accompanied by the significant downregulation of Keap1, $\operatorname{Tg} f \beta 1 / 2$, and $f n 1$, and the prominent upregulation of $N r f 2$. Therefore, the inhibition of AR and the restoration of the miR200a-3p/miR-141-3p levels may be a potential research direction for the treatment of DN.

The expression of miR-506-3p is downregulated in HGtreated HK-2 cells and the serum of patients with DN, while overexpression of miR-506-3p inhibits inflammation, OS, and pyroptosis in HG-treated HK-2 cells (125). miR-15b$5 \mathrm{p}$ is significantly decreased in HG-treated podocytes, which is accompanied by increased levels of podocyte apoptosis and OS (112). Overexpression of miR-15b-5p inhibits cell apoptosis, decreases the expression of the OS-related markers $M D A$ and NOX4, and increases the levels of SOD and hydrogen peroxide, which may occur by targeting Semaphorin $3 A$ (Sema3A) (126). miR-124a expression in bone marrow 
stromal stem cells (BMSCs) has a protective effect on OSinduced podocyte damage; transfection of BMSCs with miR124a inhibits the phosphoinositide 3-kinase/mTOR signaling pathway, thus protecting podocytes (127). This suggests that the combined effects of BMSCs and miRNA may be beneficial for the treatment of DN.

\section{IncRNAs and Diabetic Nephropathy-Related OS}

lncRNA refers to a long-chain RNA molecule with a length $>200 \mathrm{nt}$ and no protein coding ability, and regulate the process of DN. The expression of the IncRNA KCNQ1OT1 is increased in the serum of patients with DN and in HG-treated HK-2 cells (125). Further, KCNQ1OT1 directly targets miR-506-3p, and therefore, the interference of KCNQ1OT1 expression promotes miR-506-3p expression, thereby inhibiting inflammation, OS, and pyroptosis in HG-treated HK-2 cells (125). Another study in HK-2 cells showed that the expression of the lncRNA GAS5 decreases in HG-treated HK-2 cells, while the overexpression of GAS5 reduces ROS and MDA levels, and increases SOD levels (108). miRNA-452-5p expression is increased in HG-treated HK2 cells, and interference of GAS5 expression may reverse the effects of miRNA-452-5p on HG-induced inflammation, OS, and pyroptosis of renal tubular cells (108). Furthermore, lncRNA Blnc1 is highly expressed in the serum of patients with $\mathrm{DN}$, STZ-induced DN models, and HG-treated HK-2 cells; it is involved in the occurrence and development of $\mathrm{DN}$, and its interference significantly reduces renal fibrosis, inflammation, and OS (128). Wang et al. (129) found that Linc00462 is significantly upregulated in the kidneys of patients with $\mathrm{DN}$, and that its level increases in a glucose concentration- and time-dependent manner in HG-treated HK-2 and HMC cells. Knockdown of Linc00462 significantly reduces the cell viability of HG-treated cells and the levels of ROS and MDA induced by HG, while increasing the levels of SOD and catalase. Therefore, it upregulates the antioxidant system against ROS, indicating that knocking out Linc00462 may be a potential treatment for DN.

In addition to renal tubular cells, impairment of lncRNA expression in glomerular cells also participates in DN-related OS. Zhang et al. (112) found that the expression of lncRNA CASC2 decreases in the serum of patients with DN and in HGtreated HMCs, while the upregulation of CASC2 inhibits HMC proliferation, ECM accumulation, and HG-induced OS. miR$133 \mathrm{~b}$, the target of CASC2, is highly expressed in the serum of patients with DN and in HG-treated HMCs; the enrichment in miR-133b reverses the effect of CASC2 upregulation. The study confirmed that the upregulation of CASC2 inhibits HMC cell proliferation, ECM accumulation, and HG-induced OS through the miR-133b/FOXP1 axis, suggesting that CASC2 may be used as a novel target for DN treatment (112). Linc01619 is downregulated in renal tissues of patients with $\mathrm{DN}$, and is associated with proteinuria and decreased renal function (113). Further, in vitro experiments confirmed that Linc01619 is expressed in the cytoplasm of podocytes, participating in the ERS signaling pathway, where it may be used as a competitive endogenous RNA that regulates the ERS and podocyte damage mediated by miR-27a/FOXO1 in DN (113). These results indicate that lncRNAs play an important role in DN-related OS and may improve DN by affecting miRNA expression.

\section{Other ncRNAs and Diabetic Nephropathy-Related OS}

circRNA is a large ncRNA, which binds to miRNA and terminates the regulation of its target genes, namely, the circRNA-miRNAmRNA regulatory network. circLRP6 regulates HG-induced MC proliferation, OS, ECM accumulation, and inflammation by competitively binding to miR-205 and HMGB1, and activating the Toll-like receptor $4 / \mathrm{NF}-\kappa \mathrm{B}$ pathway (130). These findings provide a better understanding of the pathogenesis of DN. In addition, antisense mitochondrial non-coding RNA-2 (ASncmtRNA-2) is expressed in experimental DN models and in vitro in human renal mesangial cells (HRMCs). Furthermore, it is significantly upregulated in mice with hereditary type $2 \mathrm{DM}$ that also develop DN. When using the nitric oxide synthase inhibitor NG-nitro-L-arginine methyl ester (L-NAME) to inhibit ROS, the upregulation of ASncmtRNA-2 in DN is significantly reduced. In cultured HRMCs, HG treatment upregulates ASncmtRNA-2 expression in a time-dependent manner. Incubation of HRMCs with L-NAME also reduces the glucose-induced upregulation of ASncmtRNA-2. In addition, ROS upregulate ASncmtRNA-2 and may promote glomerular fibrosis in $\mathrm{DN}$ by actively regulating the expression of pro-fibrotic factors (131). This indicates that ASncmtRNA-2 is involved in the DN-related OS response and renal fibrosis development.

\section{POTENTIAL CLINICAL APPLICATIONS OF OS-RELATED ncRNAs IN DN}

The morbidity and mortality of DN remains high worldwide. Many studies have shown that early and timely intervention in DN significantly limits proteinuria, thereby preventing further development of DN (132). Therefore, the identification of novel molecular markers and drug targets for DN prevention and treatment is urgently needed. The application of next-generation sequencing technologies for RNAseq revealed that changes in the expression of miRNAs are very common in the pathogenesis of diabetes and DN (133). In addition, miRNAs are widely present in various parts of the cell, increasing their potential as DNspecific molecular markers.

The expression of many miRNAs related to OS is different in diabetic and healthy people $(134,135)$. For example, OS-related miR-21 has been proposed as a diagnostic marker for prediabetes (135), and receiver operating characteristic (ROC) curve analysis has shown that the expression of miR-21 is a suitable candidate marker for distinguishing diabetes from prediabetes (sensitivity, 93\%; specificity, 35\%). The ROC area under the curve (AUC) was 0.7 (Table 1). Moreover, the serum levels of miR-99b, miR-486$5 \mathrm{p}, \mathrm{miR}-122-5 \mathrm{p}$, and miR-20a in the serum, whose target genes are closely related to OS (114), are considered to have diagnostic value in diabetic kidney diseases (Table 1).

Although, ncRNAs are closely associated with DN-related OS, there are limited related clinical drug studies. Shao et al. (105) 
TABLE 1 | Diagnostic index of miRNA in human DM-related OS.

\begin{tabular}{|c|c|c|c|c|c|c|c|}
\hline miRNAs & Diseases & Sample numbers (control/diseases) & AUC & Sensitivity & Specificity & OR $\left(95 \% \mathrm{Cl}^{*}\right)$ & Ref \\
\hline miR-21 & Diabetes & $44 / 27$ & 0.7 & $93 \%$ & $35 \%$ & 1.05 (1.01-1.09) & (121) \\
\hline miR-99b & DKD & $25 / 42$ & 0.895 & - & - & - & (114) \\
\hline$m i R-486-5 p$ & DKD & $25 / 42$ & 0.853 & - & - & - & (114) \\
\hline miR-122-5p & DKD & $25 / 42$ & 0.8 & - & - & - & (114) \\
\hline miR-20a & DKD & $25 / 42$ & 0.697 & - & - & - & (114) \\
\hline
\end{tabular}

DM, diabetes mellitus; AUC, area under the curve; OR, odds ratio; Cl, confidence interval; Ref, reference; DKD, diabetic kidney disease.

treated $\mathrm{DN}$ rats with ginsenoside $\mathrm{Rb} 1$, triclosan, and ginsenoside $\mathrm{Rb} 1$ plus trigonelline and found that the combination of ginsenoside $\mathrm{Rb} 1$ and trigonelline significantly alleviates renal dysfunction, OS, and pathological changes. Further, studies have confirmed that ginsenoside $\mathrm{Rb} 1$ and trigonelline regulate the expression of miR-3550, which regulates the $\mathrm{Wnt} / \beta$-catenin signaling pathway, therefore preventing the occurrence of diabetic kidney disease (105). Sitagliptin, a dipeptidyl peptidase 4 inhibitor used for the treatment of type $2 \mathrm{DM}$, has a protective effect on diabetic chronic kidney diseases. Civantos et al. (136) conducted proteomic and miRNA transcriptomic analysis of the renal cortex of wild-type (Wistar), diabetic Goto-Kakizaki (GK) rats, and rats treated with siglitine. Proteomic analysis of diabetic GK and Wistar rats showed differential expression of 39 proteins, and significant changes occurred among 15 miRNAs in GK rats, which are mainly related to OS and catabolism. Further studies have confirmed that treatment with sitagliptin improves OS in experimental DN via the mir-200a/Keap-1/Nrf2 antioxidant pathway, thus, exerting renal-protective effects. Ochratoxin A, a mycotoxin with nephrotoxic and potentially carcinogenic activity, induces the expression of miR-200c and miR-132 in renal proximal epithelial cells. miR-200c and miR-132 target NRF2 and HO-1, respectively, thereby promoting renal OS and inducing renal injury (137). Thus, ncRNAs are considered potential therapeutic targets based on their regulatory roles. Further, research is necessary to explore the diagnostic value of OS-related ncRNAs in DN, to identify novel drug targets, and prevent DN.

\section{CONCLUSIONS}

Recently, ncRNAs have been recognized as a "new star" in the field of DN. As a class of novel regulatory molecules, they participate in multiple steps of DN by modulating the expression of several related genes. The development of RNAseq and next generation sequencing technologies has allowed the identification of a large number of ncRNAs. The extraction and detection of ncRNAs has higher specificity and sensitivity than those of proteins. Thus, ncRNAs can potentially be used as potential diagnostic and prognostic biomarkers. In addition, we can silence or activate ncRNAs in DN patients by exogenous means. For example, we can wrap the silenced or active lentiviral vector of ncRNAs into exosomes or other vehicles in vitro, and perform a targeted injection into the corresponding organs via blood. This can be developed as a therapy for DN. Considering their roles in the progression of DN-related OS, ncRNAs have great potential as "biological tools" for the screening, diagnosis, and treatment of DN, and may ultimately cure DN. However, there is a long road from the scientific research of ncRNAs to their clinical application. Recently, the research on ncRNA and DN has faced a series of challenges and limitations: (1) many ncRNAs are yet to be discovered and identified in DN through the development of RNAseq and next generation sequencing technologies; (2) ncRNAs need to be further investigated to determine whether they are specifically related to one or more diseases, and to explore the underlying molecular mechanisms whereby ncRNAs contribute to the disease; (3) The exact and specific mechanisms whereby ncRNAs regulate DN-related OS remain to be discovered; (4) According to many animal models, ncRNAs play a role in DM and its complications; however, the lack of clinical trials confirming the accuracy and safety of these findings remains an issue; (5) Many endogenous and exogenous factors involved in ncRNA production have not yet been identified, which hinders the use of ncRNAs as clinical therapeutic targets for DN treatment.

In this review, the regulatory role of ncRNAs in DNrelated OS has been summarized. These ncRNAs regulate individual target genes or constitute interaction networks, such as lncRNA-miRNA-mRNA or circRNA-miRNA-mRNA, and play an important role in the regulation of DN-related OS. As our understanding of the molecular mechanisms involved in ncRNA regulation and their function in vitro and in vivo increases, novel and more effective treatment methods will be developed, which may cure DN by targeting the corresponding key ncRNAs.

\section{AUTHOR CONTRIBUTIONS}

$\mathrm{XH}, \mathrm{GK}$, and $\mathrm{YZ}$ conceived the concept and wrote the manuscript. CO edited and improved the manuscript. All authors contributed to the article and approved the submitted version.

\section{FUNDING}

This study was supported by the National Natural Science Foundation of China (81903032 and 81860156), the China Postdoctoral Science Foundation (2020M672520), and the Youth Fund of Xiangya Hospital (2018Q011). 


\section{REFERENCES}

1. Schernthaner G, Mogensen C E, Schernthaner GH. The effects of GLP-1 analogues, DPP-4 inhibitors and SGLT2 inhibitors on the renal system. Diab Vasc Dis Res. (2014) 11:306-23. doi: 10.1177/1479164114542802

2. Zhang MH, Feng L, Zhu MM, Gu J, Jiang J, Cheng XD, et al. The antiinflammation effect of Moutan Cortex on advanced glycation end productsinduced rat mesangial cells dysfunction and High-glucose-fat diet and streptozotocin-induced diabetic nephropathy rats. J Ethonpharmaco. (2014) 151:591-600. doi: 10.1016/j.jep.2013.11.015

3. Kanwar YS, Wada J, Sun L, Xie P, Wallner EI, Chen S, et al. Diabetic nephropathy: mechanisms of renal disease progression. Exp Biol Med. (2008) 233:4-11. doi: 10.3181/0705-MR-134

4. Lin YC, Chang YH, Yang SY, Wu KW, Chu TS. Update of pathophysiology and management of diabetic kidney disease. J Formos Med Assoc. (2018) 117:662-75. doi: 10.1016/j.jfma.2018.02.007

5. Bondeva T, Wolf G. Reactive oxygen species in diabetic nephropathy: friend or foe?. Nephrol Dial Transplant. (2014) 29:1998-2003. doi: 10.1093/ndt/gfu037

6. Andresdottir G, Jensen ML, Carstensen B, Parving HH, Rossing K, Hansen TW, et al. Improved survival and renal prognosis of patients with type 2 diabetes and nephropathy with improved control of risk factors. Diabetes Care. (2014) 37:1660-7. doi: 10.2337/dc13-2036

7. Sies H. Oxidative stress: a concept in redox biology and medicine. Redox Biol. (2015) 4:180-3. doi: 10.1016/j.redox.2015.01.002

8. Singh DK, Winocour P, Farrington K. Oxidative stress in early diabetic nephropathy: fueling the fire. Nat Rev Endocrinol. (2011) 7:176-84. doi: 10.1038/nrendo.2010.212

9. Buranasin P, Mizutani K, Iwasaki K, Mahasarakham CPN, Kido D, Takeda $\mathrm{K}$, et al. High glucose-induced oxidative stress impairs proliferation and migration of human gingival fibroblasts. PLoS ONE. (2018) 13:e201855. doi: 10.1371/journal.pone.0201855

10. Chen $X$, Shen WB, Yang $P$, Dong $D$, Sun W, Yang P. High glucose inhibits neural stem cell differentiation through oxidative stress and endoplasmic reticulum stress. Stem Cells Dev. (2018) 27:745-55. doi: 10.1089/scd.2017.0203

11. Zheng DH, Han ZQ, Wang XX, MA D, Zhang J. Erythropoietin attenuates high glucose-induced oxidative stress and inhibition of osteogenic differentiation in periodontal ligament stem cell (PDLSCs). Chem Biol Interact. (2019) 305:40-7. doi: 10.1016/j.cbi.2019.03.007

12. Xiang H, Xue W, Wu X, Zheng J, Ding C, Li Y, et al. FOXP1 inhibits high glucose-induced ECM accumulation and oxidative stress in mesangial cells. Chem Biol Interact. (2019) 313:108818. doi: 10.1016/j.cbi.2019.108818

13. Das NA, Carpenter AJ, Belenchia A, Aroor AR, Noda M, Siebenlist $\mathrm{U}$, et al. Empagliflozin reduces high glucose-induced oxidative stress and miR-21-dependent TRAF3IP2 induction and RECK suppression, and inhibits human renal proximal tubular epithelial cell migration and epithelial-to-mesenchymal transition. CellSignal. (2020) 68:109506. doi: 10.1016/j.cellsig.2019.109506

14. Zhang W, Sui Y. CircBPTF knockdown ameliorates high glucose-induced inflammatory injuries and oxidative stress by targeting the miR-384/LIN28B axis in human umbilical vein endothelial cells. Mol Cell Biochem. (2020) 471:101-11. doi: 10.1007/s11010-020-03770-2

15. Pal S, Rao GN, Pal A. High glucose-induced ROS accumulation is a critical regulator of ERK1/2-Akt-tuberin-mTOR signalling in RGC-5 cells. Life Sci. (2020) 256:117914. doi: 10.1016/j.lfs.2020.117914

16. Pan X, Chen J, Wang T, Zhang M, Wang H, Gao H. Essential role of high glucose-induced overexpression of PKCbeta and PKCdelta in GLP1 resistance in rodent cardiomyocytes. Diabetes Metab Syndr Obes. (2019) 12:2289-302. doi: 10.2147/DMSO.S215789

17. Gerardo YF, Andrade-Sierra J, Pazarin-Villasenor L, Santana-Arciniega C, De Jesús TE, De Jesús Torres-VE, et al. The role of dietary antioxidants on oxidative stress in diabetic nephropathy. Iran J Kidney Dis. (2020) 14:81-94.

18. Sureshbabu A, Ryter S W, Choi ME. Oxidative stress and autophagy: crucial modulators of kidney injury. Redox Biol. (2015) 4:208-14. doi: 10.1016/j.redox.2015.0 1.001
19. Jha JC, Banal C, Chow BS, Cooper ME, Jandeleit-Dahm K. Diabetes and kidney disease: role of oxidative stress. Antioxid Redox Signal. (2016) 25:65784. doi: 10.1089/ars.2016.6664

20. Hombach S, Kretz M. Non-coding RNAs: classification, biology, and functioning. Adv Exp Med Biol. (2016) 937:317. doi: 10.1007/978-3-319-42059-2_1

21. Ou C, Sun Z, Li X, Ren W, Qin Z, Zhang X, et al. MiR-590-5p, a densitysensitive microRNA, inhibits tumorigenesis by targeting YAP1 in colorectal cancer. Cancer Lett. (2017) 399:53-63. doi: 10.1016/j.canlet.2017.04.011

22. Vishnoi A, Rani S. MiRNA biogenesis and regulation of diseases: an overview. Methods Mol Biol. (2017) 1509:110. doi: 10.1007/978-1-4939-6524-3_1

23. Jiang C, Xu D, You Z, Xu K, Tian W. Dysregulated circRNAs and ceRNA network in esophageal squamous cell carcinoma. Front Biosci. (2019) 24:27790. doi: $10.2741 / 4717$

24. Yan Q, He X, Kuang G, Ou C. CircRNA cPWWP2A: an emerging player in diabetes mellitus. J Cell Commun Signal. (2020) 14:3513. doi: 10.1007/s12079-020-00570-7

25. Khanipouyani F, Akrami H, Fattahi MR. Circular RNAs as important players in human gastric cancer. Clin Transl Oncol. (2020) 23:1021. doi: $10.1007 / \mathrm{s} 12094-020-02419-2$

26. Mourksi NE, Morin C, Fenouil T, Diaz JJ, Marcel V. snoRNAs offer novel insight and promising perspectives for lung cancer understanding and management. Cells. (2020) 9:541. doi: 10.3390/cells9030541

27. Michel CI, Holley CL, Scruggs BS, Sidhu R, Brookheart RT, Listenberger LL. Small nucleolar RNAs U32a, U33, and U35a are critical mediators of metabolic stress. Cell Metab. (2011) 14:33-44. doi: 10.1016/j.cmet.2011.04.009

28. Sun Z, Ou C, Liu J, Chen C, Zhou Q, Yang S, et al. YAP1-induced MALAT1 promotes epithelial-mesenchymal transition and angiogenesis by sponging miR-126-5p in colorectal cancer. Oncogene. (2019) 38:262744. doi: 10.1038/s41388-018-0628-y

29. Lorenzen JM, Thum T. Long noncoding RNAs in kidney and cardiovascular diseases. Nat Rev Nephrol. (2016) 12:360-73. doi: 10.1038/nrneph.2016.51

30. He X, Ou C, Xiao Y, Han Q, Li H, Zhou S. LncRNAs: key players and novel insights into diabetes mellitus. Oncotarget. (2017) 8:7132541. doi: 10.18632/oncotarget.19921

31. Aalijahan H, Ghorbian S. Long non-coding RNAs and cervical cancer. Exp Mol Pathol. (2019) 106:7-16. doi: 10.1016/j.yexmp.2018.11.010

32. He D, Zheng J, Hu J, Chen J, Wei X. Long non-coding RNAs and pyroptosis. Clin Chim Acta. (2020) 504:201-8. doi: 10.1016/j.cca.2019.11.035

33. Huang $\mathrm{S}, \mathrm{Li} \mathrm{X}$, Zheng $\mathrm{H}, \mathrm{Si} \mathrm{X}$, Li B, Wei G, et al. Loss of super-enhancer-regulated circRNA Nfix induces cardiac regeneration after myocardial infarction in adult mice. Circulation. (2019) 139:285776. doi: 10.1161/CIRCULATIONAHA.118.038361

34. Yang JX, Rastetter RH, Wilhelm D. Non-coding RNAs: an introduction. $A d v$ Exp Med Biol. (2016) 886:13-32. doi: 10.1007/978-94-017-7417-8_2

35. Ernst C, Morton C. Identification and function of long non-coding RNA. Front Cell Neurosci. (2013) 7:168. doi: 10.3389/fncel.2013.00168

36. Mercer TR, Dinger ME, Mattick JS. Long non-coding RNAs: insights into functions. Nat Rev Genet. (2009) 10:155-9. doi: 10.1038/nrg2521

37. Wu H, Yang L, Chen LL. The diversity of long noncoding RNAs and their generation. Trends Genet. (2017) 33:540-52. doi: 10.1016/j.tig.2017.05.004

38. Yao RW, Wang Y, Chen LL. Cellular functions of long noncoding RNAs. Nat Cell Biol. (2019) 21:542-51. doi: 10.1038/s41556-019-0311-8

39. Catalanotto C, Cogoni C, Zardo G. MicroRNA in control of gene expression: an overview of nuclear functions. Int J Mol Sci. (2016) 17:1712. doi: $10.3390 / \mathrm{ijms} 17101712$

40. Ma X, Cen S, Wang L, Zhang C, Wu L, Tian X, et al. Genomewide identification and comparison of differentially expressed profiles of miRNAs and lncRNAs with associated ceRNA networks in the gonads of Chinese soft-shelled turtle, Pelodiscus sinensis. BMC Genomics. (2020) 21:443. doi: 10.1186/s12864-020-06826-1

41. Prabahar A, Natarajan J. MicroRNA mediated network motifs in autoimmune diseases and its crosstalk between genes, functions, and pathways. J Immunol Methods. (2017) 440:1926. doi: $10.1016 /$ j.jim.2016.10.002 
42. He X, Li S, Yu B, Wu Y, Zhang M, He Y, et al. Up-regulation of LINC00467 promotes the tumourigenesis in colorectal cancer. J Cancer. (2019) 10:640513. doi: $10.7150 /$ jca. 32216

43. Morris KV, Mattick JS. The rise of regulatory RNA. Nat Rev Genet. (2014) 15:423-37. doi: $10.1038 / \operatorname{nrg} 3722$

44. Flynn RA, Chang HY. Long noncoding RNAs in cell-fate programming and reprogramming. Cell Stem Cell. (2014) 14:752-61. doi: 10.1016/j.stem.2014.05.014

45. Evans JR, Feng FY, Chinnaiyan AM. The bright side of dark matter: lncRNAs in cancer. J Clin Incest. (2016) 126:2775-82. doi: 10.1172/JCI84421

46. Kopp F, Mendell JT. Functional classification and experimental dissection of long noncoding RNAs. Cell. (2018) 172:393407. doi: 10.1016/j.cell.2018.01.011

47. Engreitz JM, Haines JE, Perez EM, Munson J, Chen J, Kane M, et al. Local regulation of gene expression by lncRNA promoters, transcription and splicing. Nature. (2016) 539:452-5. doi: 10.1038/nature20149

48. Dong Y, Yoshitomi T, Hu JF, Cui J. Long noncoding RNAs coordinate functions between mitochondria and the nucleus. Epigenetics Chromatin. (2017) 10:41. doi: 10.1186/s13072-017-0149-x

49. Chen LL, Yang L. Regulation of circRNA biogenesis. RNA Biol. (2015) 12:381-8. doi: 10.1080/15476286.2015.1020271

50. Kristensen LS, Andersen MS, Stagsted L, Ebbesen KK, Hansen TB, Kjems J. The biogenesis, biology, and characterization of circular RNAs. Nat Rev Genet. (2019) 20:675-91. doi: 10.1038/s41576-019-0158-7

51. Nie H, Wang Y, Liao Z, Zhou J, Ou C. The function and mechanism of circular RNAs in gastrointestinal tumours. Cell Prolif. (2020) 53:e12815. doi: $10.1111 /$ cpr.12815

52. He X, Ou C. CircRNA circHIPK3: a novel therapeutic target for angiotensin II-induced cardiac fibrosis. Int J Cardiol. (2020) 312:98. doi: 10.1016/j.ijcard.2020.03.034

53. Du WW, Zhang C, Yang W, Yong T, Awan FM, Yang B. Identifying and characterizing circRNA-protein interaction. Theranostics. (2017) 7:418391. doi: 10.7150/thno.21299

54. Li X, Yang L, Chen LL. The biogenesis, functions, and challenges of circular RNAs. Mol Cell. (2018) 71:428-42. doi: 10.1016/j.molcel.2018.06.034

55. Fan HM, Sun XY, Guo W, Zhong A, Niu W, Zhao L, et al. Differential expression of microRNA in peripheral blood mononuclear cells as specific biomarker for major depressive disorder patients. J Psychiatr Res. (2014) 59:45-52. doi: 10.1016/j.jpsychires.2014.08.007

56. Jeck WR, Sorrentino JA, Wang K, Slevin MK, Burd CE, Liu J, et al. Circular RNAs are abundant, conserved, associated with ALU repeats. RNA. (2013) 19:141-57. doi: 10.1261/rna.035667.112

57. Yang F, Chen Y, Xue Z, Lv Y, Shen L, Li K, et al. High-throughput sequencing and exploration of the IncRNA-circRNA-miRNAmRNA network in type 2 diabetes mellitus. Biomed Res Int. (2020) 2020:8162524. doi: 10.1155/2020/8162524

58. Maxwell ES, Fournier MJ. The small nucleolar RNAs. Annu Rev Biochem. (1995) 64:897-934. doi: 10.1146/annurev.bi.64.070195.004341

59. Kiss T. Small nucleolar RNAs: an abundant group of noncoding RNAs with diverse cellular functions. Cell. (2002) 109:1458. doi: 10.1016/S0092-8674(02)00718-3

60. Smith CM, Steitz JA. Sno storm in the nucleolus: new roles for myriad small RNPs. Cell. (1997) 89:669-72. doi: 10.1016/S0092-8674(00) 80247-0

61. Tycowski KT, You ZH, Graham PJ, Steitz JA. Modification of U6 spliceosomal RNA is guided by other small RNAs. Mol Cell. (1998) 2:62938. doi: 10.1016/S1097-2765(00)80161-6

62. Jady BE, Bertrand E, Kiss T. Human telomerase RNA and box H/ACA scaRNAs share a common Cajal body-specific localization signal. J Cell Biol. (2004) 164:647-52. doi: 10.1083/jcb.200310138

63. Bachellerie JP, Cavaille J, Huttenhofer A. The expanding snoRNA world. Biochimie. (2002) 84:775-90. doi: 10.1016/S0300-9084(02)01402-5

64. Shim K, Begum R, Yang C, Wang H. Complement activation in obesity, insulin resistance, and type 2 diabetes mellitus. World J Diabetes. (2020) 11:1-12. doi: 10.4239/wjd.v11.i1.1

65. Lim A. Diabetic nephropathy - complications and treatment. Int J Nephrol Renovasc Dis. (2014) 7:361-81. doi: 10.2147/IJNRD. S40172
66. Giralt-Lopez A, Molina-Van DBM, Vergara A, García-Carro C, Seron D, Jacobs-Cachá C, et al. Revisiting experimental models of diabetic nephropathy. Int J Mol Sci. (2020) 21:3587-609. doi: 10.3390/ijms21103587

67. Duran-Salgado MB, Rubio-Guerra AF. Diabetic nephropathy and inflammation. World J Diabetes. (2014) 5:393-8. doi: 10.4239/wjd.v5.i3.393

68. Qi C, Mao X, Zhang Z, Wu H. Classification and differential diagnosis of diabetic nephropathy. J Diabetes Res. (2017) 2017:8637138. doi: 10.1155/2017/8637138

69. Macisaac R J, Ekinci EI, Jerums G. Markers of and risk factors for the development and progression of diabetic kidney disease. Am J Kidney Dis. (2014) 63:S39-62. doi: 10.1053/j.ajkd.2013.10.048

70. Roa V, LVB Roa, Tan SH, Candasamy M, Bhattamisra SK. Diabetic nephropathy: an update on pathogenesis and drug development. Diabetes Metab Syndr. (2019) 13:754-62. doi: 10.1016/j.dsx.2018.11.054

71. Aghadavod E, Khodadadi S, Baradaran A, Nasri P, Bahmani M, RafieianKopaei M. Role of oxidative stress and inflammatory factors in diabetic kidney disease. Iran J Kidney Dis. (2016) 10:337-43.

72. Khodaeian M, Enayati S, Tabatabaei-Malazy O, Amoli MM. Association between genetic variants and diabetes mellitus in iranian populations: a systematic review of observational studies. J Diabetes Res. (2015) 2015:585917. doi: 10.1155/2015/585917

73. Rizvi S, Raza ST, Mahdi F. Association of genetic variants with diabetic nephropathy. World J Diabetes. (2014) 5:809-16. doi: 10.4239/wjd.v5.16.809

74. Stefanidis I, Tziastoudi M, Tsironi EE, Dardiotis E, Tachmitzi SV, Fotiadou $\mathrm{A}$, et al. The contribution of genetic variants of SLC2A1 gene in T2DM and T2DM-nephropathy: association study and meta-analysis. Ren Fail. (2018) 40:561-76. doi: 10.1080/0886022X.2018.1496931

75. Raptis AE, Viberti G. Pathogenesis of diabetic nephropathy. Exp Clin Endocrinal Diabetes. (2001) 109 (Suppl. 2):S42437. doi: 10.1055/s-2001-18600

76. Bleyer AJ, Fumo P, Snipes ER, Simmons DA, Ziyadeh FN. Polyol pathway mediates high glucose-induced collagen synthesis in proximal tubule. Kidney Int. (1994) 45:659-66. doi: 10.1038/ki.1994.88

77. Yang PY, Li PC, Feng B. Protective effects of gliclazide on high glucose and AGEs-induced damage of glomerular mesangial cells and renal tubular epithelial cells via inhibiting RAGE-p22phox-NF-kB pathway. Eur Rev Med Pharmacol Sci. (2019) 23:9099-107. doi: 10.26355/eurrev_201910_19313

78. Goh SY, Cooper ME. Clinical review: the role of advanced glycation end products in progression and complications of diabetes. J Clin Endocrinol Metab. (2008) 93:1143-52. doi: 10.1210/jc.2007-1817

79. Wada J, Makino H. Inflammation and the pathogenesis of diabetic nephropathy. Clin Sci. (2013) 124:139-52. doi: 10.1042/CS20120198

80. Lv N, Li C, Liu X, Qi C, Wang Z. MiR-34b alleviates high glucoseinduced inflammation and apoptosis in human HK-2 cells via IL6R/JAK2/STAT3 signaling pathway. Med Sci Monit. (2019) 25:814251. doi: 10.12659/MSM.917128

81. Chen F, Zhu X, Sun Z, Ma Y. Astilbin inhibits high glucose-induced inflammation and extracellular matrix accumulation by suppressing the TLR4/MyD88/NF-kappaB pathway in rat glomerular mesangial cells. Front Pharmacol. (2018) 9:1187. doi: 10.3389/fphar.2018.01187

82. Brosius FR. Trophic factors and cytokines in early diabetic glomerulopathy. Exp Diabesty Res. (2003) 4:225-33. doi: 10.1155/EDR.2003.225

83. Kashihara N, Haruna Y, Kondeti VK, Kanwar YS. Oxidative stress in diabetic nephropathy. Curr Med Chem. (2010) 17:4256-69. doi: 10.2174/092986710793348581

84. Zhang XM, Wang YZ, Tong JD, Ning XC, Zhou FQ, Yang XH, et al. Pyruvate alleviates high glucose-induced endoplasmic reticulum stress and apoptosis in HK-2 cells. Febs Open Bio. (2020) 10:82734. doi: 10.1002/2211-5463.12834

85. Liu H, Sun HL. LncRNA TCF7 triggered endoplasmic reticulum stress through a sponge action with miR-200c in patients with diabetic nephropathy. Eur Rev Med Pharmacol Sci. (2019) 23:5912-22. doi: 10.26355/eurrev_201907_18336

86. Mizushima N, Komatsu M. Autophagy: renovation of cells and tissues. Cell. (2011) 147:728-41. doi: 10.1016/j.cell.2011.10.026

87. Kume S, Yamahara K, Yasuda M, Maegawa H, Koya D. Autophagy: emerging therapeutic target for diabetic nephropathy. Semin Nephrol. (2014) 34:916. doi: 10.1016/j.semnephrol.2013.11.003 
88. Kume S, Thomas MC, Koya D. Nutrient sensing, autophagy, diabetic nephropathy. Diabetes. (2012) 61:23-9. doi: 10.2337/db11-0555

89. Yang D, Livingston MJ, Liu Z, Dong G, Zhang M, Chen $\mathrm{JK}$, et al. Autophagy in diabetic kidney disease: regulation, pathological role and therapeutic potential. Cell Mol Life Sci. (2018) 75:669-88. doi: 10.1007/s00018-017-2639-1

90. Tanaka Y, Kume S, Kitada M, Kanasaki K, Uzu T, Maegawa H, et al. Autophagy as a therapeutic target in diabetic nephropathy. Exp Diabetes Res. (2012) 2012:628978. doi: 10.1155/2012/628978

91. Gudehithlu KP, Garcia-Gomez I, Vernik J, Brecklin C, Kraus M, Cimbaluk DJ, et al. In diabetic kidney disease urinary exosomes better represent kidney specific protein alterations than whole urine. Am J Nephrol. (2015) 42:418-24. doi: 10.1159/000443539

92. Barutta F, Tricarico M, Corbelli A, Annaratone L, Pinach S, Grimaldi S, et al. Urinary exosomal microRNAs in incipient diabetic nephropathy. PLoS ONE. (2013) 8:e73798. doi: 10.1371/journal.pone.0073798

93. Elmarakby AA, Sullivan JC. Relationship between oxidative stress and inflammatory cytokines in diabetic nephropathy. Cardiovasc Ther. (2012) 30:49-59. doi: 10.1111/j.1755-5922.2010.00218.x

94. Rochette L, Zeller M, Cottin Y, Vergely C. Diabetes, oxidative stress, and therapeutic strategies. Biochim Biophys Acta. (2014) 1840:270929. doi: 10.1016/j.bbagen.2014.05.017

95. Wolf G. New insights into the pathophysiology of diabetic nephropathy: from haemodynamics to molecular pathology. Eur J Clin Invest. (2004) 34:785-96. doi: 10.1111/j.1365-2362.2004.01429.x

96. Ighodaro OM. Molecular pathways associated with oxidative stress in diabetes mellitus. Biomed Pharmacother. (2018) 108:656-62. doi: 10.1016/j.biopha.2018.09.058

97. Duni A, Liakopoulos V, Roumeliotis S, Peschos D, Dounousi E. Oxidative stress in the pathogenesis and evolution of chronic kidney disease: untangling ariadne's thread. Int J Mol Sci. (2019) 20:3711. doi: 10.3390/ijms20153711

98. Gnudi L, Coward R, Long DA. Diabetic nephropathy: perspective on novel molecular mechanisms. Trends Endocrinol Metab. (2016) 27:82030. doi: 10.1016/j.tem.2016.07.002

99. Wei PZ, Szeto CC. Mitochondrial dysfunction in diabetic kidney disease. Clin Chim Acta. (2019) 496:108-16. doi: 10.1016/j.cca.2019.07.005

100. Su H, Wan C, Song A, Qiu Y, Xiong W, Zhang C. Oxidative stress and renal fibrosis: mechanisms and therapies. Adv Exp Med Biol. (2019) 1165:585604. doi: 10.1007/978-981-13-8871-2_29

101. Chhabra R, Adlakha YK, Hariharan M, Scaria V, Saini N. Upregulation of miR-23a-27a-24-2 cluster induces caspase-dependent and -independent apoptosis in human embryonic kidney cells. PLOS ONE. (2009) 4:e5848. doi: 10.1371/journal.pone.0005848

102. Chhabra R, Dubey R, Saini N. Gene expression profiling indicate role of ER stress in miR-23a 27a 24-2 cluster induced apoptosis in HEK293T cells. RNA Biol. (2011) 8:648-64. doi: 10.4161/rna.8.4.15583

103. Cadenas S. Mitochondrial uncoupling, ROS generation and cardioprotection. Biochism Biophsy Acta Bioenerg. (2018) 1859:940-50. doi: 10.1016/j.bbabio.2018.05.019

104. Wei W, Peng J, Shen T. Rosuvastatin alleviates ischemia/reperfusion injury in cardiomyocytes by downregulating Hsa-miR-24-3p to target upregulated uncoupling protein 2. Cell Reprogram. (2019) 21:99-107. doi: 10.1089/cell.2018.0039

105. Shao X, Chen C, Miao C, Yu X, Li X, Geng J, et al. Expression analysis of microRNAs and their target genes during experimental diabetic renal lesions in rats administered with ginsenoside Rb1 and trigonelline. Pharmazie. (2019) 74:492-8. doi: 10.1691/ph.2019.8903

106. Song J, Zhang $\mathrm{H}$, Sun $\mathrm{Y}$, Guo $\mathrm{R}$, Zhong $\mathrm{D}$, $\mathrm{Xu} \mathrm{R}$, et al. Omentin-1 protects renal function of mice with type 2 diabetic nephropathy via regulating miR-27a-Nrf2/Keap1 axis. Biomed Pharmacother. (2018) 107:440-6. doi: 10.1016/j.biopha.2018.0 8.002

107. Muratsu-Ikeda S, Nangaku M, Ikeda $\mathrm{Y}$, Tanaka T, Wada T, Inagi R. Downregulation of miR-205 modulates cell susceptibility to oxidative and endoplasmic reticulum stresses in renal tubular cells. PLoS ONE. (2012) 7:e41462. doi: 10.1371/journal.pone. 0041462
108. Xie $\mathrm{C}, \mathrm{Wu} \mathrm{W}$, Tang $\mathrm{A}$, Tanaka $\mathrm{T}$, Wada $\mathrm{T}$, Inagi J. IncRNA GAS5/miR-452-5p reduces oxidative stress and pyroptosis of highglucose-stimulated renal tubular cells. Dabetes Metab Syndr Obes. (2019) 12:2609-17. doi: 10.2147/DMSO.S228654

109. Wang Q, Wang Y, Minto AW, Wang J, Shi Q, Li X, et al. MicroRNA377 is up-regulated can lead to increased fibronectin production in diabetic nephropathy. Faseb J. (2008) 22:4126-35. doi: 10.1096/fj.08-112326

110. Bai XY, Ma Y, Ding R, Fu B, Shi S, Chen X. MiR-335 M and miR-34a Promote renal senescence by suppressing mitochondrial antioxidative enzymes. J Am Soc Nephrol. (2011) 22:1252-61. doi: 10.1681/ASN.2010040367

111. Kato M, Putta S, Wang M, Yuan H, Lanting L, Nair I, et al. TGF-beta activates Akt kinase through a microRNA-dependent amplifying circuit targeting PTEN. Nat Cell Biol. (2009) 11:881-9. doi: 10.1038/ncb1897

112. Zhang XL, Zhu HQ, Zhang Y, Zhang CY, Jiao JS, Xing XY. LncRNA CASC2 regulates high glucose-induced proliferation, extracellular matrix accumulation and oxidative stress of human mesangial cells via miR-133b/FOXP1 axis. Eur Rev Med Pharmacol Sci. (2020) 24:802-12. doi: 10.26355/eurrev_202001_20063

113. Bai X, Geng J, Li X, Wan J, Liu J, Zhou Z, et al. Long noncoding RNA LINC01619 regulates microRNA-27a/Forkhead box protein $\mathrm{O} 1$ and endoplasmic reticulum stress-mediated podocyte injury in diabetic nephropathy. Antioxid Redox Signal. (2018) 29:355-76. doi: 10.1089/ars.2017.7278

114. Regmi A, Liu G, Zhong X, Hu S, Ma R, Gou L, et al. Evaluation of serum microRNAs in patients with diabetic kidney disease: a nested casecontrolled study and bioinformatics analysis. Med Sci Monit. (2019) 25:1699708. doi: 10.12659/MSM.913265

115. Zhou L, Xu D-y, Sha W-g, Shen L, Lu GY, Wang MJ, et al. High glucose induces renal tubular epithelial injury via Sirt1/NFkappaB/microR-29/Keap1 signal pathway. J Transl Med. (2015) 13:352. doi: 10.1186/s12967-015-0710-y

116. Yang S, Fei X, Lu Y, Xu B, Ma Y, Wan H. MiRNA-214 suppresses oxidative stress in diabetic nephropathy via the ROS/Akt/mTOR signaling pathway and uncoupling protein 2. Exp Ther Med. (2019) 17:353038. doi: 10.3892/etm.2019.7359

117. Li H, Wang D, Chen Y, Yang M. Beta-caryophyllene inhibits high glucose-induced oxidative stress, inflammation, and extracellular matrix accumulation in mesangial cells. Int Immunopharmacol. (2020) 84:106556. doi: 10.1016/j.intimp.2020.106556

118. Dong C, Wu G, Li H, Qiao Y, Gao S. Ampelopsin inhibits high glucoseinduced extracellular matrix accumulation and oxidative stress in mesangial cells through activating the Nrf2/HO-1 pathway. Phytother Res. (2020) 34:2044-52. doi: 10.1002/ptr.6668

119. Setyowati KD, Sepramaniam S, Tan HZ, Armugam A, Jeyaseelan miR$25 \mathrm{~K}$, and miR-92a regulate insulin I biosynthesis in rats. RNA Biol. (2013) 10:1365-78. doi: 10.4161/rna.25557

120. Fu Y, Zhang Y, Wang Z, Wang L, Wei X, Zhang B, et al. Regulation of NADPH oxidase activity is associated with miRNA-25-mediated NOX4 expression in experimental diabetic nephropathy. Am J Nephrol. (2010) 32:581-9. doi: 10.1159/000322105

121. Varga ZV, Kupai K, Szucs G, Gáspár R, Pálóczi J, Faragó N, et al. MicroRNA-25-dependent up-regulation of NADPH oxidase 4 (NOX4) mediates hypercholesterolemia-induced oxidative/nitrative stress and subsequent dysfunction in the heart. J Mol Cell Cardiol. (2013) 62:111-21. doi: 10.1016/j.yjmcc.2013.05.009

122. Li H, Zhu X, Zhang J, Shi J. MicroRNA-25 inhibits high glucose-induced apoptosis in renal tubular epithelial cells via PTEN/AKT pathway. Biomed Pharmacother. (2017) 96:471-479. doi: 10.1016/j.biopha.2017.10.019

123. Wan RJ, Li YH. MicroRNA146a/NAPDH oxidase4 decreases reactive oxygen species generation and inflammation in a diabetic nephropathy model. $\mathrm{Mol}$ Med Rep. (2018) 17:4759-66. doi: 10.3892/mmr.2018.8407

124. Wei J, Zhang Y, Luo Y, Guo R, Zhong D, Xu R, et al. Aldose reductase regulates $\mathrm{miR}-200 \mathrm{a}-3 \mathrm{p} / 141-3 \mathrm{p}$ to coordinate Keap 1Nrf2, Tgfbeta1/2, and Zeb1/2 signaling in renal mesangial cells and the renal cortex of diabetic mice. Free Radic Biol Med. (2014) 67:91-102. doi: 10.1016/j.freeradbiomed.2013.10.811

125. Zhu B, Cheng X, Jiang Y, Cheng M, Chen L, Bao J, et al. Silencing of KCNQ1OT1 decreases oxidative stress and pyroptosis of renal 
tubular epithelial cells. Diabetes Metab Syndr Obes. (2020) 13:36575. doi: 10.2147/DMSO.S225791

126. Fu Y, Wang C, Zhang D, Chu X, Zhang Y, Li J. MiR-15b-5p ameliorated high glucose-induced podocyte injury through repressing apoptosis, oxidative stress, and inflammatory responses by targeting Sema3A. J Cell Physiol. (2019) 234:20869-78. doi: 10.1002/jcp.28691

127. Sun J, Lv J, Zhang W, Li L, Geng Y, Yin A. Combination with miR-124a improves the protective action of BMSCs in rescuing injured rat podocytes from abnormal apoptosis and autophagy. J Cell Biochem. (2018) 119:716676. doi: $10.1002 / j c b .26771$

128. Feng X, Zhao J, Ding J, Shen X, Zhou J, Xu Z. LncRNA Blnc1 expression and its effect on renal fibrosis in diabetic nephropathy. Am J Transl Res. (2019) 11:5664-72.

129. Wang R, Yan Y, Li C. LINC00462 is involved in high glucose-induced apoptosis of renal tubular epithelial cells via AKT pathway. Cell Bil Int. (2019) 44:286-94. doi: 10.1002/cbin.11231

130. Chen B, Li Y, Liu Y, Xu Z. CircLRP6 regulates high glucose-induced proliferation, oxidative stress, ECM accumulation, and inflammation in mesangial cells. J Cell Physiol. (2019) 234:21249-59. doi: 10.1002/jcp.28730

131. Gao Y, Chen ZY, Wang Y, Liu Y, Ma JX, Li YK. Long non-coding RNA ASncmtRNA-2 is upregulated in diabetic kidneys and high glucose-treated mesangial cells. Exp Ther Med. (2017) 13:581-7. doi: 10.3892/etm.2017.4027

132. Paul P, Chakraborty A, Sarkar D, Langthasa M, Rahman M, Singha RS, et al. Interplay between miRNAs and human diseases. J Cell Physiol. (2018) 233:2007-18. doi: 10.1002/jcp.25854

133. Lee JH, Gao C, Peng G, Greer C, Ren S, Wang Y, et al. Analysis of transcriptome complexity through RNA sequencing in normal and failing murine hearts. Circ Res. (2011) 109:133241. doi: 10.1161/CIRCRESAHA.111.249433
134. Shihana F, Joglekar MV, Raubenheimer J, Hardikar AA, Buckley NA, Seth D. Circulating human microRNA biomarkers of oxalic acid-induced acute kidney injury. Arch Toxical. (2020) 94:1725-37. doi: 10.1007/s00204-020-02679-5

135. La Sala L, Mrakic-Sposta S, Tagliabue E, Prattichizzo F, Micheloni S, Sangalli E, et al. Circulating microRNA-21 is an early predictor of ROS-mediated damage in subjects with high risk of developing diabetes and in drug-naive T2D. Cardiovasc Diabetol. (2019) 18:18. doi: 10.1186/s12933-019-0824-2

136. Civantos E, Bosch E, Ramirez E, Zhenyukh O, Egido J, Lorenzo O, et al. Sitagliptin ameliorates oxidative stress in experimental diabetic nephropathy by diminishing the miR-200a/Keap-1/Nrf2 antioxidant pathway. Diabetes Metab Syndr Obes. (2017) 10:207-22. doi: 10.2147/DMSO.S132537

137. Stachurska A, Ciesla M, Kozakowska M, Wolffram S, Rimbach G, Jozkowicz A, et al. Cross-talk between microRNAs, nuclear factor E2-related factor 2 , and heme oxygenase-1 in ochratoxin A-induced toxic effects in renal proximal tubular epithelial cells. Mol Nutr Food Res. (2013) 57:50415. doi: $10.1002 / \mathrm{mnfr} .201200456$

Conflict of Interest: The authors declare that the research was conducted in the absence of any commercial or financial relationships that could be construed as a potential conflict of interest.

Copyright $\odot 2021 \mathrm{He}$, Kuang, Zuo, Li, Zhou and Ou. This is an open-access article distributed under the terms of the Creative Commons Attribution License (CC BY). The use, distribution or reproduction in other forums is permitted, provided the original author(s) and the copyright owner(s) are credited and that the original publication in this journal is cited, in accordance with accepted academic practice. No use, distribution or reproduction is permitted which does not comply with these terms. 